\title{
BMJ Open Sex-specific association of serum uric acid dynamics with the incidence of metabolic syndrome in a health check- up Chinese population: a prospective cohort study
}

\author{
Ying Liu, ${ }^{1}$ Yongjun Fan, ${ }^{2}$ Qigui Liu, ${ }^{2}$ Kehua Liu, ${ }^{2}$ Fangfang Chen, ${ }^{2}$ Xiao Tang, ${ }^{2}$ \\ Guorong Li, ${ }^{2}$ Dongmei Hu, ${ }^{2}$ Guirong Song (D) ${ }^{2}$
}

To cite: Liu Y, Fan Y, Liu Q, et al. Sex-specific association of serum uric acid dynamics with the incidence of metabolic syndrome in a health check-up Chinese population: a prospective cohort study. BMJ Open 2020;10:e035289. doi:10.1136/ bmjopen-2019-035289

- Prepublication history for this paper is available online. To view these files, please visit the journal online (http://dx.doi. org/10.1136/bmjopen-2019035289).

YL, YF and QL contributed equally.

Received 26 0ctober 2019 Revised 07 February 2020 Accepted 01 June 2020

Check for updates

(c) Author(s) (or their employer(s)) 2020. Re-use permitted under CC BY-NC. No commercial re-use. See rights and permissions. Published by BMJ.

${ }^{1}$ The Physical Examination Centre of the Second Affiliated Hospital, Dalian Medical University, Dalian, Liaoning,

China

${ }^{2}$ Department of Health Statistics, School of Public Health, Dalian Medical University, Dalian, Liaoning, China

Correspondence to

Guirong Song;

songsara110@163.com

\section{ABSTRACT}

Objective Many studies have demonstrated that elevated serum uric acid (SUA) level is linked with metabolic syndrome (MetS). However, whether there is a sex difference in the association between SUA and MetS has not been determined. This study aimed to accurately explore the impact of SUA longitudinal changes on MetS by sex.

Design A prospective cohort study.

Setting The Health Check-up Centre of the Second Hospital affiliated with Dalian Medical University from 2010 to 2016.

Participants A health check-up cohort of 577 men and 1698 women aged 20-60 years who did not exhibit MetS or hyperuricaemia at baseline and underwent at least two physical examinations from 2010 to 2016.

Primary and secondary outcome measures Weight, height, blood pressure and blood biochemistry parameters, including SUA, were measured. MetS was defined according to the Joint Interim Statement criteria.

Methods Based on longitudinal data, a linear mixed-effects model was constructed to explore the characteristics of SUA dynamic changes in males and females, and joint modelling of longitudinal and survival data was done to analyse the association between SUA dynamic changes and MetS occurrence.

Results The natural logarithm of SUA (LNSUA) in females exhibited a gradually increasing trend, and its annual growth rate in females who developed MetS was greater than that of the non-MetS females. The longitudinal growth of SUA in females was a risk factor for the onset of MetS, and the estimated HR was 13.2580 (95\% Cl 1.9106 to 91.9957) for each 1-unit rise in LNSUA longitudinally. An association between the longitudinal growth of LNSUA and MetS was not found in males.

Conclusions The longitudinal increase in SUA in females could increase the risk of MetS, even if the SUA changes within the normal range. The longitudinal increase in SUA in males was not a predictor for MetS.

\section{INTRODUCTION}

Metabolic syndrome (MetS) is generally defined as a cluster of cardiovascular risk
Strengths and limitations of this study

- This study is the first to explore the association of serum uric acid (SUA) dynamics with the incidence of metabolic syndrome (MetS) based on longitudinal data from a Chinese population.

- This study monitored the SUA levels longitudinally before the occurrence of MetS and performed joint modelling of longitudinal and survival data to completely and accurately quantify the predictive effects of changes in SUA over time on the risk of developing MetS.

- The primary limitations of this study were the short follow-up period and the lack of sufficient information on medication use, dietary habits and other lifestyle factors.

factors, including abdominal obesity, hyperglycaemia, high blood pressure (BP), high triglycerides (TG) and low high-density lipoprotein cholesterol (HDL-C). ${ }^{1}$ It has been powerfully demonstrated that MetS can increase the risks of cardiovascular disease (CVD), type 2 diabetes mellitus and certain cancers. ${ }^{2-4}$ Moreover, the prevalence of MetS worldwide is approximately $10 \%-40 \%$ and is continuously increasing. ${ }^{5}$ Therefore, MetS has become a major public health challenge. Preventing the occurrence and development of MetS early is a favourable way to address this challenge. Meanwhile, the prevention strategy of MetS is inseparable from the search for biomarkers and risk factors of MetS in the early stage.

Uric acid (UA) is the end oxidation product of purine metabolism in humans. In the recent years, serum UA (SUA), as a predictive biomarker and risk factor of MetS, has received much attention from researchers, like other classical risk factors (eg, glycaemia, 
TG and HDL-C).$^{6-11}$ Many studies have demonstrated that elevated SUA level is linked with hypertension, diabetes, obesity, insulin resistance and MetS. ${ }^{6-14}$ However, the results of these studies are somewhat controversial. In particular, whether there is a sex difference in the association between SUA and MetS or its components has not been determined. Some studies found a stronger association between SUA level and MetS in women than in men. ${ }^{15-18}$ Some studies found that SUA level could predict the risk of MetS only in women. ${ }^{19}$ However, some studies found that there was no sex difference in the risk of MetS caused by high SUA level. ${ }^{20}$

These inconsistent results may be due to study populations of different ages and races. In addition, most previous studies were cross-sectional surveys, with only a few prospective cohort studies. It is common to mainly use traditional statistical methods (logistic models or Cox models) to analyse the effects of baseline SUA level on MetS. Individual SUA level may change over time because of changes in diet, disease and medication. Obviously, traditional statistical analysis cannot examine whether the SUA level has changed during the follow-up or whether its change may have influenced the study results. To clearly understand the effect of dynamic changes in SUA on the incidence risk of MetS, we applied a more powerful method, joint modelling of longitudinal and survival data (JMLS), which consists of two submodels, a measurement model for longitudinal data and an intensity model describing the time to event. ${ }^{21}$ To our knowledge, no one has evaluated the effect of the longitudinal increments in SUA activities over time on the incidence risk of MetS by JMLS until now.

\section{MATERIAL AND METHODS Design and subjects}

The study population was selected from subjects who underwent two health checkups at the Second Hospital Affiliated with Dalian Medical University of China from March 2010 to April 2016. Data from each subject's initial health examination were defined as the baseline data. Initially, a total of 2747 subjects were included based on the following criteria: (1) Subjects were between 20 and 60 years old at baseline, (2) Subjects did not have a history of hypertension, diabetes, CVD, viral hepatitis, liver cirrhosis, autoimmune liver disease, renal disease or rheumatic disease at baseline, (3) Subjects did not have missing data related to MetS components such as height, weight, systolic BP (SBP), diastolic BP (DBP), fasting plasma glucose (FPG), TG or HDL-C at baseline, (4) Subjects had not been diagnosed with MetS at baseline, (5) Subjects did not have missing measurements of SUA at baseline and had normal SUA levels (UA $\leq 420 \mu \mathrm{mol} / \mathrm{L}$ in males, UA $\leq 360 \mu \mathrm{mol} / \mathrm{L}$ in females ${ }^{22}$ ) and (6) Subjects underwent at least two physical examinations from March 2010 to April 2016. Next, 503 subjects who had missing data related to MetS components or SUA measurements or who were diagnosed with CVD, viral hepatitis, liver cirrhosis, autoimmune liver disease, renal disease or rheumatic disease during the follow-up were excluded from the study. Finally, 2244 subjects were included in our study. Once MetS occurred for a subject, his or her follow-up was terminated.

\section{Anthropometric and laboratory measurements}

The subjects underwent routine physical examinations, including measurements of weight, height, BP and blood biochemistry parameters, including TG, HDL-C, FPG and SUA. All these measuring methods have been formally described elsewhere. ${ }^{23}$

\section{Definition of MetS}

The Joint Interim Statement criteria in 2009 were used to diagnose MetS. ${ }^{24}$ As waist circumference (WC) was not obtained in the health check-up, body mass index (BMI) was used as a substitute for WC to evaluate obesity because it is strongly correlated with WC in patients with MetS. ${ }^{25}$ Subjects were diagnosed with MetS if they had three or more of the following risk factors: (1) obesity: BMI $\geq 25 \mathrm{~kg} / \mathrm{m}^{2} ;{ }^{26}$ (2) elevated BP (antihypertensive drug treatment in a patient with a history of hypertension was an alternate indicator): $\mathrm{SBP} \geq 130 \mathrm{~mm} \mathrm{Hg}$ and/ or DBP $\geq 85 \mathrm{~mm} \mathrm{Hg}$; (3) elevated TG (drug treatment for increased TG was an alternate indicator): $\geq 150 \mathrm{mg} /$ dL $(1.7 \mathrm{mmol} / \mathrm{L})$; (4) reduced HDL-C (drug treatment for reduced HDL-C was an alternate indicator): $<40 \mathrm{mg}$ / $\mathrm{dL}(1.0 \mathrm{mmol} / \mathrm{L})$ in males, $<50 \mathrm{mg} / \mathrm{dL}(1.3 \mathrm{mmol} / \mathrm{L})$ in females and (5) elevated FPG (drug treatment of elevated glucose was an alternate indicator): $\geq 100 \mathrm{mg} / \mathrm{dL}$.

\section{Explanation for variables}

A list of variables used in the model with an explanation for each is shown in table 1 . The dependent variable SUA was converted to natural logarithm of SUA (LNSUA) to satisfy the requirement of a normal distribution of JMLS. In order to explain the implication of the intercept in the model, the subject's baseline age was centralised by subtracting the median of the baseline ages.

\begin{tabular}{ll}
\hline Table 1 & Explanation of variables used in analyses \\
\hline Variables & Explanation \\
\hline Time & $\begin{array}{l}\text { The interval between each health examination } \\
\text { date and the first health examination date in } \\
\text { years }\end{array}$ \\
Age & $\begin{array}{l}\text { The baseline age after centralisation in years } \\
\text { State }\end{array}$ \\
ons if MetS, 0 if no MetS \\
LNSUA-0 & $\begin{array}{l}\text { The SUA level after natural-logarithmic } \\
\text { conversion }\end{array}$ \\
& $\begin{array}{l}\text { The baseline SUA level after natural- } \\
\text { logarithmic conversion }\end{array}$ \\
\hline
\end{tabular}

LNSUA, natural logarithm of SUA; MetS, metabolic syndrome; SUA, serum uric acid. 


\section{Statistical analysis}

Data were expressed as the median and IQR for nonnormally distributed variables and were compared by the Wilcoxon rank sum test. Categorical data were described as proportions.

Given the large difference between the numbers of males and females in our study and the difference in SUA levels between males and females, a linear mixed-effects model was constructed to explore the characteristics of SUA dynamic changes in males and females.

The basic linear mixed-effects model was

$$
y_{i j}=b_{00}+\mu_{0 i}+\left(b_{10}+\mu_{1 i}\right) \text { time }+b_{2} \text { age }+b_{3} \text { state }+\varepsilon_{i j}
$$

In this model, $y_{i j}$ denotes the observed LNSUA value for the $i^{\text {th }}(i=1,2 \ldots n)$ subject at the $j^{t h}(j=0,1 \ldots, t)$ follow-up time point; $b_{00}, b_{10}, b_{2}, b_{3}$ are the fixed effects and denote the average of the intercept, the regression coefficient of measured time points (time), the centralised baseline age (age), and the state of MetS (including MetS or nonMetS), respectively; $\mu_{0 i}, \mu_{1 i}$ are the random effects of intercept and the slope of time, and we used their standard deviations $(\sigma)$ to describe them; and $\varepsilon_{i j}$ is the total error of the model, which was measured using the following equation: $\varepsilon_{i j} \sim N\left(0, \sigma^{2}\right)$. We also determined the effects of the interactions between these independent variables, such as time:age, time:state and age:state.

JMLS was constructed to assess the impact of timevarying LNSUA on the hazard for MetS in males and females. The basic JMLS is specified as:

$$
\left\{\begin{array}{l}
y_{i j}=m_{i}(t)+\varepsilon_{i j}=b_{0 i}+b_{1 i} t_{i j}+\varepsilon_{i j}=b_{00}+u_{0 i}+\left(b_{10}+u_{1 i}\right) t_{i j}+\varepsilon_{i j} \\
h_{i}\left\{t \mid M_{i}(t), x_{i}\right\}=h_{0}(t) \exp \left\{\sum \beta_{i} x_{i}+\alpha m_{i}(t)\right\}
\end{array}\right.
$$

The first part is the linear mixed-effects model for LNSUA as noted above. In the second part, the survival submodel, $h_{i}(t)$ denotes the hazard function for the $i^{t h}$ subject with covariates, $M_{i}(t)$ and $x_{i}$, at time $t$. The baseline hazard is given by $h_{0}(t) ; M_{i}(t)$ is the whole longitudinal history of the marker levels up to time $t ; m_{i}(t)$ denotes the true value of $y_{i j} ; x_{i}$ represents a certain time-independent covariate of $h_{i}(t) ; \beta_{i}$ denotes the regression coefficient of $x_{i}$; and $\alpha$ stands for the association parameter assessing the relationship between the longitudinal measurement and the survival submodel.

The basic descriptive analysis was performed by SPSS V.24.0 software (IBM), and $\mathrm{p}<0.05$ was considered statistically significant. The linear mixed-effects model and JMLS were conducted using the R V.3.2.3 package $\mathrm{JM}^{27}$ to account for baseline and longitudinal correlations between LNSUA and MetS.

\section{Patient and public involvement statement}

The patients, the public and any third parties were not involved in our research.

\section{RESULTS}

\section{Basic characteristics}

A total of 2244 subjects aged 20-60 years at baseline, with a median age of 36 years, consisted of 546 men (24.33\%) with a median age of 39 and 1698 women $(75.67 \%)$ with a median age of 34 . The average follow-up time was 3.9 $(2.1-4.9)$ years, $3.0(2.0-4.0)$ years for males and 3.9 $(2.3-5.0)$ years for females. During the follow-up period, 273 subjects developed MetS, including 112 men and 161 women. The total cumulative incidence of MetS was $12.17 \%$, and it was $20.51 \%$ in males and $9.48 \%$ in females. The total incidence density was 3.44 per 100 person-years, and it was 6.32 per 100 person-years in males and 2.62 per 100 person-years in females.

The average level of SUA in the MetS group in each follow-up year was generally higher than that in the nonMetS group regardless of sex (table 2). As we can see intuitively from figure 1, the average SUA level of males or females with MetS basically increased with time, but that in the female non-MetS group remained more or less flat during the follow-up period.

\section{Longitudinal changes in LNSUA}

The results of the linear mixed-effects model in males are shown in table 3. The LNSUA level in males was significantly affected by time $(\mathrm{p}<0.0001)$ but was not significantly affected by age or state. The average level of LNSUA at baseline was $5.7943 \mu \mathrm{mol} / \mathrm{L}$ for a 39 (the median of the baseline age)-year-old man who did not develop MetS during the follow-up. The average level of LNSUA at baseline in males who developed MetS was the same as that of the non-MetS males. The LNSUA level in males significantly increased with the passing of each follow-up year, and the annual growth rate of LNSUA was $0.0123 \mu \mathrm{mol} / \mathrm{L}$ on average. In addition, none of the interactions was statistically significant in the male model, so males who developed MetS exhibited a similar average growth rate of LNSUA as the non-MetS males.

The results of the linear mixed-effects model in females are shown in table 4 . The LNSUA level in females was significantly affected by time $(\mathrm{p}<0.0001)$ and state $(p<0.05)$ but was not significantly affected by age. The average level of LNSUA at baseline was $5.4661 \mu \mathrm{mol} / \mathrm{L}$ for a 34 (the median of the baseline age)-year-old woman who did not develop MetS during the follow-up. The average level of LNSUA in females who developed MetS was $0.0380 \mu \mathrm{mol} / \mathrm{L}$ higher than that of the non-MetS females. The annual growth rate of LNSUA level in the non-MetS females was $0.0103 \mu \mathrm{mol} / \mathrm{L}$ on average. Moreover, the interaction between time and state was statistically significant in the female model, so the annual growth rate of LNSUA in females who developed MetS was $0.0179 \mu \mathrm{mol} / \mathrm{L}$ greater than that of the non-MetS females.

\section{Association between SUA dynamics and MetS}

As we can see from table 5 , the survival submodel in males showed that LNSUA-0 and age did not have a significant impact on the incidence of MetS $(\mathrm{p}>0.05)$. Furthermore, the association parameter $\alpha$ was not statistically significant, which meant that there was no correlation between changes in LNSUA and MetS for males. 
Table 2 Baseline characteristics of male and female subjects according to the development of MetS

\begin{tabular}{|c|c|c|c|c|c|c|}
\hline & \multicolumn{3}{|c|}{ Male group } & \multicolumn{3}{|c|}{ Female group } \\
\hline & MetS & Non-MetS & P value* & MetS & Non-MetS & $\begin{array}{l}\text { P } \\
\text { value* }\end{array}$ \\
\hline $\begin{array}{l}\text { SBP }(m m \\
\mathrm{Hg})\end{array}$ & 126.0 (117.0 to 133.0$)$ & $123.0(115.0$ to 130.0$)$ & 0.111 & 120.0 (110.0 to 127.0$)$ & $114.0(107.0,122.0)$ & $<0.001$ \\
\hline $\begin{array}{l}\mathrm{DBP}(\mathrm{mm} \\
\mathrm{Hg})\end{array}$ & $76.0(70.0,82.0)$ & 74.0 (68.0 to 81.0$)$ & 0.111 & 73.0 (66.0 to 80.0$)$ & 70.0 (63.0 to 76.0$)$ & $<0.001$ \\
\hline $\begin{array}{l}\text { FPG } \\
(\mathrm{mmol} / \mathrm{L})\end{array}$ & 5.4 (5.2 to 5.6$)$ & 5.4 (5.1 to 5.6$)$ & 0.059 & 5.2 (4.9 to 5.5$)$ & 5.2 (4.9 to 5.5$)$ & 0.463 \\
\hline $\mathrm{TG}(\mathrm{mmol} / \mathrm{L})$ & $1.2(0.9$ to 1.6$)$ & $1.0(0.8$ to 1.2$)$ & $<0.001$ & $0.9(0.7$ to 1.2$)$ & $0.8(0.6$ to 1.0$)$ & $<0.001$ \\
\hline $\begin{array}{l}\text { HDL-C } \\
(\mathrm{mmol} / \mathrm{L})\end{array}$ & $1.2(1.0$ to 1.3$)$ & $1.3(1.1$ to 1.5$)$ & $<0.001$ & $1.4(1.2$ to 1.5$)$ & $1.5(1.3$ to 1.7$)$ & $<0.001$ \\
\hline
\end{tabular}

Data are median $\left(\mathrm{P}_{25}, \mathrm{P}_{75}\right)$.

* $P$ value was determined by the Wilcoxon rank sum test between the MetS group and the non-MetS group in males or females.

BMI, body mass index; DBP, diastolic blood pressure; FPG, fasting plasma glucose; HDL-C, high-density lipoprotein cholesterol; MetS, metabolic syndrome; SBP, systolic blood pressure; SUA, serum uric acid; TG, triglycerides.

In table 6, similarly, the survival submodel in females showed that LNSUA- 0 and age did not have a significant impact on the incidence of MetS ( $>00.05)$. However, the association parameter $\alpha$ was statistically significant, which indicated a strong positive association between longitudinal increments in LNSUA and the risk of developing MetS for females. The results implied that a 1-unit longitudinal increment in LNSUA represented a 13.2580-fold (95\% CI 1.9106 to 91.9957) increase in the risk of MetS for females.

Table 7 shows the HRs of MetS that correspond to different longitudinal increments in SUA for different SUA baseline values in females. For instance, considering the estimated parameter $\alpha=13.2580$ in the survival submodel, a 5-unit increase in SUA over the baseline SUA value of $100,200,300,360$ or 420 represented a



Figure 1 Changes in the trend of average level of SUA during follow-up in male and female according to the development of MetS. MetS, metabolic syndrome; SUA, serum uric acid. 1.1344-fold, 1.0659-fold, 1.0436-fold, 1.0363-fold or 1.0311-fold increase in the HR of MetS, respectively. Therefore, for a particular unit increment in SUA, there was a larger impact on MetS risk when starting from a lower baseline SUA level. Increments of $30 \%, 50 \%$ or $80 \%$ from a $100 \mu \mathrm{mol} / \mathrm{L}$ baseline SUA resulted in a HR of approximately $1.9701,2.8518$ or 4.5685 , respectively. However, for the same percentage increments in SUA, the HRs were the same across different baseline SUA values. In conclusion, for females, the greater the dynamic increment in SUA, the greater the incidence risk of developing MetS, regardless of the baseline SUA level.

Table 3 Dynamic changes in LNSUA by linear mixedeffects model in the male group

\begin{tabular}{lrlrr}
\hline Variable & \multicolumn{1}{l}{ B } & SE & \multicolumn{1}{l}{ Z value } & P value \\
\hline Intercept & 5.7943 & 0.0078 & 743.3294 & $<0.0001$ \\
\hline Time & 0.0123 & 0.0019 & 6.5353 & $<0.0001$ \\
Age & -0.0009 & 0.0008 & -1.0541 & 0.2923 \\
State & 0.0235 & 0.0173 & 1.3571 & 0.1753 \\
Time:age & 0.0001 & 0.0002 & 0.3889 & 0.6974 \\
Time:state & 0.0080 & 0.0049 & 1.6592 & 0.0973 \\
Age:state & 0.0002 & 0.0017 & 0.1150 & 0.9085 \\
\hline & 0.1416 & & & \\
$\sigma_{b 00}$ & & & & \\
$\sigma_{b 10}$ & 0.0156 & & & \\
$\varepsilon$ & 0.0953 & & & \\
\hline
\end{tabular}

LNSUA, natural logarithm of serum uric acid. 
Table 4 Dynamic changes in LNSUA by the linear mixedeffects model in the female group

\begin{tabular}{lrlrr}
\hline Variable & \multicolumn{1}{l}{ B } & SE & Z value & P value \\
\hline Intercept & 5.4661 & 0.0048 & 1146.9063 & $<0.0001$ \\
Time & 0.0103 & 0.0012 & 8.9604 & $<0.0001$ \\
Age & -0.0009 & 0.0005 & -1.6645 & 0.0962 \\
State & 0.0380 & 0.0156 & 2.4427 & 0.0147 \\
Time:age & 0.0001 & 0.0001 & 0.7830 & 0.4337 \\
Time:state & 0.0179 & 0.0043 & 4.1784 & $<0.0001$ \\
age:state & 0.0016 & 0.0016 & 1.0495 & 0.2941 \\
$\sigma_{b 00}$ & 0.1553 & & & \\
& & & & \\
$\sigma_{b 10}$ & 0.0118 & & & \\
$\varepsilon$ & 0.1303 & & & \\
\hline
\end{tabular}

LNSUA, natural logarithm of serum uric acid.

\section{DISCUSSION}

In this longitudinal study of MetS-free subjects with normal SUA level initially, we explored the characteristics of longitudinal changes in SUA level and the effect of longitudinal changes in SUA level on the incidence of MetS for each sex. The LNSUA level in females exhibited a gradually increasing trend, and the annual growth rate of LNSUA in females who developed MetS was greater than that of the non-MetS females. Moreover, the longitudinal growth of SUA in females was a risk factor for new onset of MetS. The same was not true males. The males who developed MetS showed a similar annual growth rate of LNSUA as the non-MetS males. Furthermore, an association between the longitudinal growth of SUA and MetS in males was not found. These new findings, which were not available from previous traditional research, could provide new insights into the risks of MetS and lay a foundation for future studies.

UA is the end catabolic product of purine from exogenous dietetic compounds as well as endogenous purine, and the SUA level results from the equilibrium between its generation, mainly in the liver, and its elimination, mostly through the kidneys. ${ }^{28}$ Theoretically, the individual SUA level keeps relatively stable due to the balance of production and excretion of UA under normal conditions. However, the individual SUA level may be elevated by various factors, such as dietary intake of high-purine foods, drinking alcohol and other unhealthy lifestyles. ${ }^{22}$ At the same time, renal hypofunction and various metabolic disorders that come with age can increase the UA produced by liver cells. As our results showed, the LNSUA level of both non-MetS male and female individuals presented a slowly increasing trend year by year during the follow-up, which illustrated that the SUA levels of healthy individuals, which are affected by various factors that are very difficult to determine, such as diet, genetics and environmental factors, ${ }^{29}$ are not constant. Thus, longitudinal measurements of SUA for an individual more truly reflect the situation of metabolism than one measurement. Furthermore, we found that the annual growth rate of LNSUA in females who developed MetS was greater than that of the nonMetS females in the linear mixed-effects model. That is, LNSUA increased faster year by year in females who developed MetS than non-MetS females. We can speculate that before the occurrence of MetS in females, the continuous growth of SUA due to various reasons could lead to excess accumulation of SUA that can further lead to vascular endothelial dysfunction, inflammation,

Table 5 The association between longitudinal LNSUA values and the incidence of MetS in the male group by JMLS

\begin{tabular}{|c|c|c|c|c|c|c|}
\hline Variable & B & SE & $Z$ value & $P$ value & $\mathbf{R R}$ & RR $95 \%$ Cl \\
\hline \multicolumn{7}{|c|}{ Longitudinal submodel fixed effects } \\
\hline Intercept & 5.7991 & 0.0071 & 829.3699 & $<0.0001$ & & \\
\hline Age & -0.0008 & 0.0008 & -1.0471 & 0.2951 & & \\
\hline Time:age & -0.0001 & 0.0003 & -0.2265 & 0.8208 & & \\
\hline \multirow{2}{*}{\multicolumn{7}{|c|}{0.1396}} \\
\hline & & & & & & \\
\hline$\sigma_{b 10}$ & 0.0186 & & & & & \\
\hline$\varepsilon$ & 0.0952 & & & & & \\
\hline$\alpha$ & 1.9809 & 1.4657 & 1.3515 & 0.1765 & 7.2493 & $(0.4099$ to 128.2113$)$ \\
\hline
\end{tabular}

JMLS, joint modelling of longitudinal and survival; LNSUA, natural logarithm of serum uric acid; MetS, metabolic syndrome; RR, risk ratio. 
Table 6 The association between longitudinal LNSUA values and the incidence of MetS in the female group by JMLS

\begin{tabular}{|c|c|c|c|c|c|c|}
\hline Variable & B & SE & $Z$ value & $P$ value & $\mathbf{R R}$ & RR $95 \% \mathrm{Cl}$ \\
\hline \multicolumn{7}{|c|}{ Longitudinal submodel fixed effects } \\
\hline Intercept & 5.4702 & 0.0046 & 1190.0323 & $<0.0001$ & & \\
\hline Time & 0.0109 & 0.0016 & 6.8320 & $<0.0001$ & & \\
\hline Age & -0.0005 & 0.0005 & -0.9702 & 0.3320 & & \\
\hline Time:age & -0.0001 & 0.0002 & -0.5320 & 0.5947 & & \\
\hline
\end{tabular}

Random effects

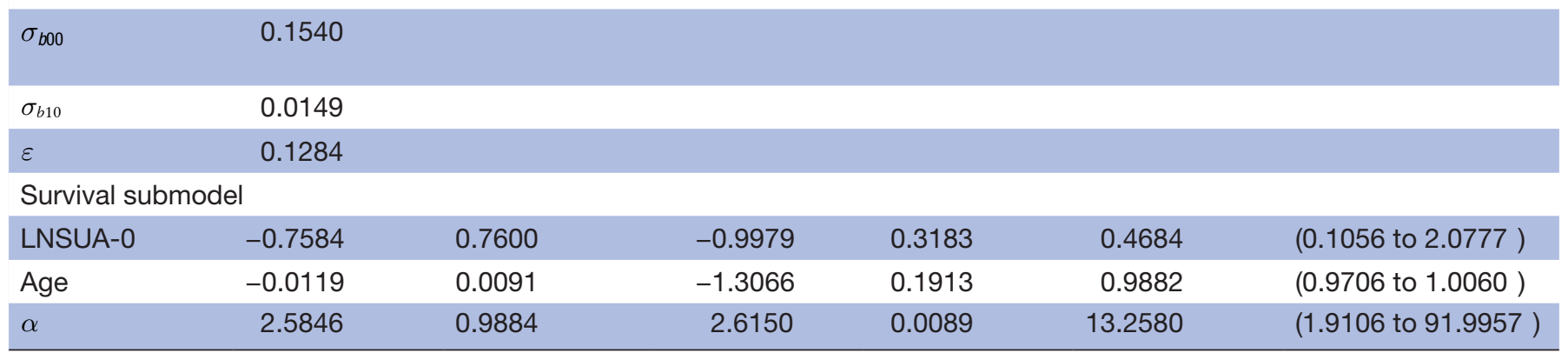

JMLS, joint modelling of longitudinal and survival; LNSUA, natural logarithm of serum uric acid; MetS, metabolic syndrome; RR, risk ratio.

nitric oxide production impairment and atherosclerosis ${ }^{30}$ ultimately resulting in the occurrence of MetS. All of the above hint that longitudinal increases in SUA in a female individual could be a precursor or a predictive biomarker of the incidence of MetS.

Moreover, in the JMLS, the association parameter $\alpha$ presented a strong positive association between longitudinal increments in LNSUA and the risk of developing MetS for women, and a 1-unit longitudinal increment in LNSUA led to a 13.2580-fold risk of MetS for females. One other point worth emphasising was that the baseline LNSUA was not significant when considering the effects of longitudinal increments in LNSUA level on MetS in the JMLS for females. This demonstrated that the effect generated by longitudinal increments of SUA on the occurrence of MetS was greater than that generated by baseline SUA in females. As we can see from table 7, a $50 \%$ increase in SUA over time for a female individual compared with its baseline level represented a 2.8518-fold increased risk of MetS. This result means that the greater the increase in the SUA level, the greater the occurrence risk of MetS for a female individual, regardless of what her SUA level at baseline is (even the level within normal reference range $(\leq 360$ $\mu \mathrm{mol} / \mathrm{L}))$.

Unlike in females, the annual growth rate of LNSUA in males who developed MetS was similar to that of the non-MetS males in the linear mixed-effects model. Interestingly, neither the baseline LNSUA level nor the longitudinal increment in LNSUA presented significant effects on the occurrence of MetS in the JMLS in males. This means that SUA for a male individual could not predict the incidence of MetS. In short, our findings by a novel powerful method, JMLS, based on a follow-up study, indicated that the longitudinal increment in SUA could predict the risk of MetS only in females. Our findings are consistent with some previous studies, for instance, by Yang et $a l^{16}$ and by Obeidat $e t a l{ }^{19} \mathrm{~A}$

Table 7 Estimated HRs of MetS for different longitudinal increases in SUA at specific SUA baseline levels for women under JMLS

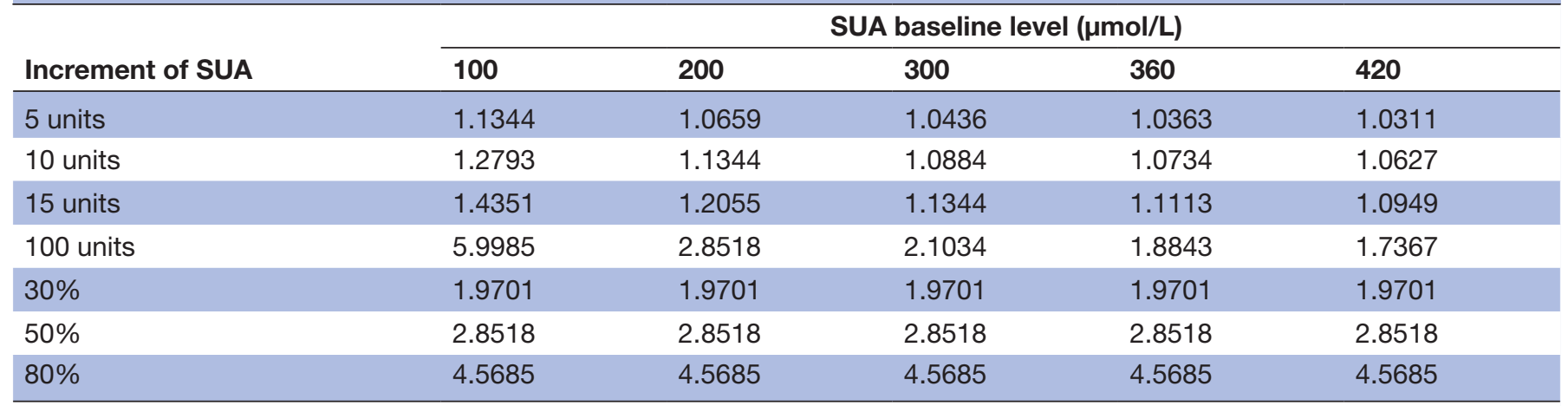

JMLS, joint modelling of longitudinal and survival; MetS, metabolic syndrome; SUA, serum uric acid. 
possible mechanism of sex differences in the relationship between SUA level and MetS is possibly due to its effects on the kidneys, and the interaction between oestrogen and other hormones has been suggested as a possible cause for sex differences. ${ }^{31}$

To date, there is no consensus on whether there is a sex difference in the association between SUA and MetS or its components. ${ }^{15-20}$ The diverse results may be ascribed to different ethnicities, sample sizes, covariates included, research designs and statistical models in the studies. Nonetheless, our findings are more realistic because they came from a joint model for longitudinal measurements of SUA and survival time to MetS, which is more advanced than traditional prediction models such as the Cox model. ${ }^{32}$ As discussed previously, the SUA level of the individuals exhibited a trajectory of growth during the follow-up, especially in females before the occurrence of MetS. However, the Cox model, a kind of static model, includes only the baseline variables but not the longitudinal history, so such a model cannot clearly reveal how the strength of the association between SUA and MetS varies over time. Instead, for prediction in the longitudinal context, JMLS, a dynamic prediction model, is able to incorporate in the model the most recent clinical history, such as the longitudinal changes in biomarkers and the incidence of a certain event. From a statistical perspective, JMLS in this study fully considered the longitudinal increments in SUA, the association of repeated measurements and the random effects among individuals. ${ }^{23}$ These are considerations the traditional analysis could not have achieved.

From the perspective of prevention, our findings benefit from discovering the signs of metabolic abnormalities as soon as possible. Because longitudinal increments in SUA are more potent predictors for the occurrence of MetS in females than the baseline SUA level, female individuals and their primary care physicians should pay more attention to dynamic changes in SUA during continuous years of physical examinations instead of only focusing on the measurement of SUA at one specific point in time. That is, when SUA levels for a female present a certain longitudinal increasing trend over time, even if the levels are within the normal reference range, the trend may herald a metabolism disturbance in the body and an increased risk of MetS. At this point, the primary care physician should help the woman take some measures (such as avoiding the intake of high-purine foods, exercising frequently) to contain the increasing trend of SUA and keep its levels stable to reduce the risk of MetS.

Several strengths of this study deserve comment. This was a longitudinal study that monitored the SUA levels longitudinally before the occurrence of MetS and applied a dynamic statistical model, so our study allows us to completely and accurately quantify the predictive effects of changes in SUA over time on the risk of developing MetS. Moreover, our findings are beneficial for identifying asymptomatic individuals at high risk for
MetS and may guide the planning and implementation of interventions for early prevention, detection, and even treatment.

However, there were still some limitations in this study. First, the subjects were only from a certain health check-up centre, and the sample size was not large enough, especially the male group. In addition, those younger than 40 at baseline made up a large proportion (51.8\% in men, $66.5 \%$ in women). These points are likely to limit generalisation of our results to the general population. Second, $37.0 \%$ of the subjects underwent three or more follow-up visits in the study. The accuracy of the results may have decreased due to the short follow-up period. Third, we only saw the linearly varying trend of SUA over time because the follow-up time was not long enough. With longer follow-up, the longitudinal trajectory of SUA may be fitted by a more complex model and presented more clearly. Finally, the present study did not collect sufficient information on medication use, dietary habits and other lifestyle factors, so there was no discussion of the other factors that could lead to the changes in SUA levels, except for baseline age and sex. Since the present study only emphasised the predictive effects of changes in SUA over time on the risk of developing MetS, these factors would unlikely account for all of the observed relationship between SUA and MetS. Further studies should have a larger sample size and a relatively long follow-up period to further validate the predictive effect of changes in SUA over time on the risk of developing MetS and its sex difference.

In conclusion, the longitudinal increase in SUA level in female individuals over time will increase the relative risk of MetS. Even if the SUA value changes within the normal range, it will still increase the relative risk of MetS. However, the longitudinal increase in SUA value in male individuals is not a predictive factor for MetS. Therefore, the dynamic changes in individual SUA levels for many years should be monitored, and early intervention should be carried out to maintain the stability of SUA levels and to effectively prevent the occurrence of MetS, especially in women.

Acknowledgements The authors thank all the study participants and the health workers who participated in the health checkups at the physical examination centre of the Second Affiliated Hospital to Dalian Medical University.

Contributors All authors meet the ICMJE criteria for authorship.YL contributed to the acquisition of the data and designed the study. GQL designed the study. YF, KL, $\mathrm{FC}, \mathrm{XT}, \mathrm{GL}$ and DH screened and extracted the data. YF performed the statistical analysis. GS reviewed the results, interpreted the data and wrote the manuscript. All authors read and approved the final manuscript.

Funding The authors have not declared a specific grant for this research from any funding agency in the public, commercial or not-for-profit sectors.

Competing interests None declared.

Patient and public involvement Patients and/or the public were not involved in the design, or conduct, or reporting, or dissemination plans of this research.

Patient consent for publication Not required.

Ethics approval All research procedures in this study were in accordance with the ethical standards of institutions and with the 1964 Declaration of Helsinki and 
its subsequent amendments. The study was approved by the institutional ethics committee of Dalian Medical University. Since it excluded all identifiable persona information, the committee waived the requirement for informed consent of the study.

Provenance and peer review Not commissioned; externally peer reviewed.

Data availability statement Data are available on reasonable request. The computing codes are available for replication by contacting corresponding authors.

Open access This is an open access article distributed in accordance with the Creative Commons Attribution Non Commercial (CC BY-NC 4.0) license, which permits others to distribute, remix, adapt, build upon this work non-commercially, and license their derivative works on different terms, provided the original work is properly cited, appropriate credit is given, any changes made indicated, and the use is non-commercial. See: http://creativecommons.org/licenses/by-nc/4.0/.

ORCID iD

Guirong Song http://orcid.org/0000-0001-6784-8805

\section{REFERENCES}

1 Engin A. The definition and prevalence of obesity and metabolic syndrome. Adv Exp Med Biol 2017;960:1-17.

2 Kastorini C-M, Panagiotakos DB, Georgousopoulou EN, et al. Metabolic syndrome and 10-year cardiovascular disease incidence: the Attica study. Nutr Metab Cardiovasc Dis 2016;26:223-31.

3 Shin J-A, Lee J-H, Lim S-Y, et al. Metabolic syndrome as a predictor of type 2 diabetes, and its clinical interpretations and usefulness. $J$ Diabetes Investig 2013;4:334-43.

4 Osaki Y, Taniguchi S-ichi, Tahara A, et al. Metabolic syndrome and incidence of liver and breast cancers in Japan. Cancer Epidemiol 2012;36:141-7.

5 Grundy SM. Metabolic syndrome update. Trends Cardiovasc Med 2016;26:364-73

6 Borges RL, Ribeiro AB, Zanella MT, et al. Uric acid as a factor in the metabolic syndrome. Curr Hypertens Rep 2010;12:113-9.

7 Kanbay M, Jensen T, Solak Y, et al. Uric acid in metabolic syndrome: from an innocent bystander to a central player. Eur J Intern Med 2016;29:3-8.

8 Lee Y-J, Cho S, Kim SR. A possible role of serum uric acid as a marker of metabolic syndrome. Intern Med J 2014;44:1210-6.

9 Cicero AFG, Fogacci F, Giovannini M, et al. Serum uric acid predicts incident metabolic syndrome in the elderly in an analysis of the Brisighella heart study. Sci Rep 2018;8:11529.

10 Yuan H, Yu C, Li X, et al. Serum uric acid levels and risk of metabolic syndrome: a dose-response meta-analysis of prospective studies. $J$ Clin Endocrinol Metab 2015;100:4198-207.

11 Kawamoto R, Ninomiya D, Kasai Y, et al. Baseline and changes in serum uric acid independently predict 11-year incidence of metabolic syndrome among community-dwelling women. J Endocrinol Invest 2018;41:959-68.

12 Sung K-C, Byrne CD, Ryu S, et al. Baseline and change in uric acid concentration over time are associated with incident hypertension in large Korean cohort. Am J Hypertens 2017;30:42-50.

$13 \mathrm{Xu} \mathrm{Y-L,} \mathrm{Xu} \mathrm{K-F,} \mathrm{Bai} \mathrm{J-L,} \mathrm{et} \mathrm{al.} \mathrm{Elevation} \mathrm{of} \mathrm{serum} \mathrm{uric} \mathrm{acid} \mathrm{and}$ incidence of type 2 diabetes: a systematic review and meta-analysis. Chronic Dis Transl Med 2016;2:81-91.
14 Yin X, Zhou J, Yu D, et al. [The correlation between serum uric acid level and abdominal obesity or metabolic syndrome]. Zhonghua Nei Ke Za Zhi 2014;53:13-18.

15 Sui X, Church TS, Meriwether RA, et al. Uric acid and the development of metabolic syndrome in women and men. Metabolism 2008;57:845-52.

16 Yang T, Chu C-H, Bai C-H, et al. Uric acid level as a risk marker for metabolic syndrome: a Chinese cohort study. Atherosclerosis 2012;220:525-31.

17 Babio N, Martínez-González MA, Estruch R, et al. Associations between serum uric acid concentrations and metabolic syndrome and its components in the PREDIMED study. Nutr Metab Cardiovasc Dis 2015;25:173-80.

18 Chiou W-K, Wang M-H, Huang D-H, et al. The relationship between serum uric acid level and metabolic syndrome: differences by sex and age in Taiwanese. J Epidemiol 2010;20:219-24.

19 Obeidat AA, Ahmad MN, Haddad FH, et al. Leptin and uric acid as predictors of metabolic syndrome in jordanian adults. Nutr Res Pract 2016;10:411-7.

20 Fu Y-Q, Yang H, Zheng J-S, et al. Positive association between metabolic syndrome and serum uric acid in Wuhan. Asia Pac J Clin Nutr 2017;26:343-50.

21 Asar Özgür, Ritchie J, Kalra PA, et al. Joint modelling of repeated measurement and time-to-event data: an introductory tutorial. Int $J$ Epidemiol 2015;44:334-44.

22 Liu L, Lou S, Xu K, et al. Relationship between lifestyle choices and hyperuricemia in Chinese men and women. Clin Rheumatol 2013;32:233-9.

23 Sun H, Liu Q, Wang X, et al. The longitudinal increments of serum alanine aminotransferase increased the incidence risk of metabolic syndrome: a large cohort population in China. Clin Chim Acta 2019;488:242-7.

24 Alberti KGMM, Eckel RH, Grundy SM, et al. Harmonizing the metabolic syndrome: a joint interim statement of the International diabetes Federation Task force on epidemiology and prevention; National heart, lung, and blood Institute; American heart association; world heart Federation; international atherosclerosis Society; and international association for the study of obesity. Circulation 2009;120:1640-5.

25 Gierach M, Gierach J, Ewertowska M, et al. Correlation between body mass index and waist circumference in patients with metabolic syndrome. ISRN Endocrinol 2014;2014:1-6.

26 Society EPoMSoCD. Recommendations on metabolic syndrome of Chinese diabetes society. Chin J Diab 2004;12:156-61.

27 Rizopoulos D. JM : an $R$ Package for the joint modelling of longitudinal and time-to-event data. J Stat Softw 2010;35:1-33.

28 Maiuolo J, Oppedisano F, Gratteri S, et al. Regulation of uric acid metabolism and excretion. Int J Cardiol 2016;213:8-14.

29 Hediger MA, Johnson RJ, Miyazaki H, et al. Molecular physiology of urate transport. Physiology 2005;20:125-33.

30 Johnson RJ, Kang D-H, Feig D, et al. Is there a pathogenetic role for uric acid in hypertension and cardiovascular and renal disease? Hypertension 2003;41:1183-90.

31 Nakagawa T, Hu H, Zharikov $\mathrm{S}$, et al. A causal role for uric acid in fructose-induced metabolic syndrome. Am J Physiol Renal Physiol 2006;290:F625-31.

$32 \mathrm{Li} \mathrm{L,} \mathrm{Luo} \mathrm{S,} \mathrm{Hu} \mathrm{B,} \mathrm{et} \mathrm{al.} \mathrm{Dynamic} \mathrm{prediction} \mathrm{of} \mathrm{renal} \mathrm{failure} \mathrm{using}$ longitudinal biomarkers in a cohort study of chronic kidney disease. Stat Biosci 2017;9:357-78. 